\title{
Osteoarthritis: An Overview.
}

\author{
Shirsa Udgata ${ }^{1}$ \\ ${ }^{I}$ (Department of Biotechnology, MGM's College of Engineering and Technology affiliated under University of \\ Mumbai, Mumbai.)
}

\begin{abstract}
Osteoarthritis (OA), also known as degenerative joint disease, is the most common form of arthritis and a leading cause of disability worldwide. The incidence of $O A$ increases with age, and disproportionately affects women. ${ }^{[I]}$ Osteoarthritis causes the cartilage in a joint to become stiff and lose its elasticity, making it more susceptible to damage. Over time, the cartilage may wear away in some areas, greatly decreasing its ability to act as a shock absorber. As the cartilage deteriorates, tendons and ligaments stretch, causing pain. If the condition worsens, the bones could rub against each other. The disease most commonly affects the middleaged and elderly, although younger people may be affected as a result of injury or overuse. Age is the strongest predictor of the disease and therefore increasing age and extended life expectancy will result in a greater occurrence of the disease. Patients affected by this disease suffer from pain and loss of function. ${ }^{[l]}$ The various treatments available are not capable of regenerating the muscle tissue back and decreasing the intensity of the disease hence; a new method called PRP (Platelet-rich Plasma) is nowadays being implemented.
\end{abstract}

Keywords: Osteoarthritis, aging, prevention, degenerative disease, PRP treatment

\section{Introduction}

There are more than 100 different types of arthritis. The most common type of arthritis is osteoarthritis (OA) or degenerative joint disease. Osteoarthritis (OA) is estimated to affect 630 million people worldwide $15 \%$ of all the people on the globe. And the need for new and ever more effective osteoarthritis treatments will continue to expand as populations' age. It is a common chronic, progressive musculoskeletal disorder characterized by gradual loss of articular cartilage. The disease most commonly affects the middle-aged and elderly, although it may begin earlier as a result of injury or overuse. It is often more painful in weight bearing joints such as the knee, hip, and spine than in the wrist, elbow, and shoulder joints. All joints may be more affected if they are used extensively in work or sports, or if they have been damaged from fractures or other injuries.

OA is regarded as a complex disease whose cause is not completely understood. Furthermore, effective biomarkers, diagnostic aids and imaging technology are not available to assist in the management of OA. There are also several areas where information is still lacking; these include: epidemiology, pathophysiology, environmental risk factors, genetic predisposition and lifestyle factors.

At present, there is no cure for OA. The management of OA is broadly divided into non-pharmacological, pharmacological, and surgical treatments. Surgical management is generally reserved for failed medical management where functional disability affects a patient's quality of life. Pharmacological management includes control of pain and improvement in function and quality of life while limiting drug toxicity. Experts in this field suggest that appropriate therapy for OA combines one or more pharmacological agents with exercise, weight loss and physical therapy (i.e. non-pharmacological therapy).

There are a number of drugs under development for symptomatic and disease modification and several studies are also evaluating alternative therapies. There are several drugs on the market whose clinical effectiveness and long-term safety still need to be determined. This assessment is especially important since OA requires longterm disease management and the disease primarily affects people over the age of 60 who are most prone to drug toxicity, and for whom the potential for drug interactions are high. Information on the impact of the disease to society and the cost of disease management (including pharmacological and non-pharmacological treatments) needs to be re-evaluated. Finally, most experts emphasize that more research efforts need to be directed towards new diagnostics, biomarkers and imaging technology. This is an essential area of research in OA since it will help to determine who is likely to get OA; severity and progression of disease; patient response to drugs, and the development of disease modifying drugs that have the potential to halt or reverse the disease. ${ }^{[2]}$

\section{Causes of OA}

1. Age: Age is the strongest risk factor for OA. Although OA can start in young adulthood, in these cases, it is often due to joint injury. Incidences of osteoarthritis increase with age due to simple "wear and tear" on the 
joints - the older you are, the more you have used them. However, that doesn't mean OA is an inevitable part of aging because not everyone gets it. ${ }^{[3]}$

1.1. Epidemiology: OA is the most common joint disorder in the world and one of the most common sources of pain and disability in the elderly. The incidence of OA is predicted to increase as the senior population grows, placing a significant financial burden on healthcare providers and governments. OA affects at least 27 million Americans and is the leading cause of disability in the United States. Compared to only $7.6 \%$ of those $18-44$ years of age and $29.8 \%$ of those $45-64$ years of age, $50 \%$ of individuals older than 65 years are diagnosed with this disease. OA affects one in six adults, and by 2030 it is estimated that $20 \%$ of people in Europe and the United States will suffer from OA. The senior population is growing rapidly in many Asian countries. It is estimated that the $65+$ population in Asia will more than double in the next two decades, increasing from $6.8 \%$ in 2008 to $16.2 \%$ in 2040 . In most of the developed world demographic change is a gradual progress following the steady socioeconomic growth over several decades. In contrast, this change is compressed into 2-3 decades in many Asian countries. For example, during the period between 2008 and 2040, it is estimated that the 65+ population will increase by $316 \%$ in Singapore, $274 \%$ in India, 269\% in Malaysia, 261\% in Bangladesh, and $256 \%$ in the Philippines. In 2008, Japan had the world's oldest population (people $65+$ represented $21.6 \%$ of whole population), and both China and India were ranked top two for the size of $65+$ population (106 and 60 million, resp.). The high prevalence and heavy impact on working capacity make OA a major social issue. Therefore, healthcare and socioeconomics need to put a high priority to the prevention and treatment of OA.

1.2. Chondrocyte Changes: The primary function of chondrocytes is to maintain cartilage homeostasis, in part through the production of extracellular matrix components. With age, chondrocytes exhibit features similar to senescent phenotypes, including telomere shortening and increased senescence-associated $\beta$-galactosidase activity. These age-related changes impair the ability of chondrocytes to maintain the surrounding extracellular matrix. Accordingly, in aged chondrocytes, synthetic activity is decreased and proteoglycans are smaller and more irregular. A reduction in the number of chondrocytes was observed in normal articular cartilage during aging, comparing with a greater loss of chondrocytes in OA cartilage, but the extent of cell death is debatable. A study showed $30 \%$ drop in cell density in human hip joint cartilage between the ages of 30 and 70 . However, a study on human knees found less than $5 \%$ cell loss during aging. Loss of the chondrocytes can be attributed to increased chondrocyte death and/or apoptosis. Although many studies reported apoptotic chondrocytes in OA cartilage, few have examined apoptosis in cartilage with normal aging, except for one study on rat cartilage. ${ }^{[4]}$

2. Heredity: Some people have an inherited defect in one of the genes responsible for making cartilage. This causes defective cartilage, which leads to more rapid deterioration of joints. People born with joint abnormalities are more likely to develop osteoarthritis, and those born with an abnormality of the spine (such as scoliosis or curvature of the spine) are more likely to develop osteoarthritis of the spine. ${ }^{[5]}$

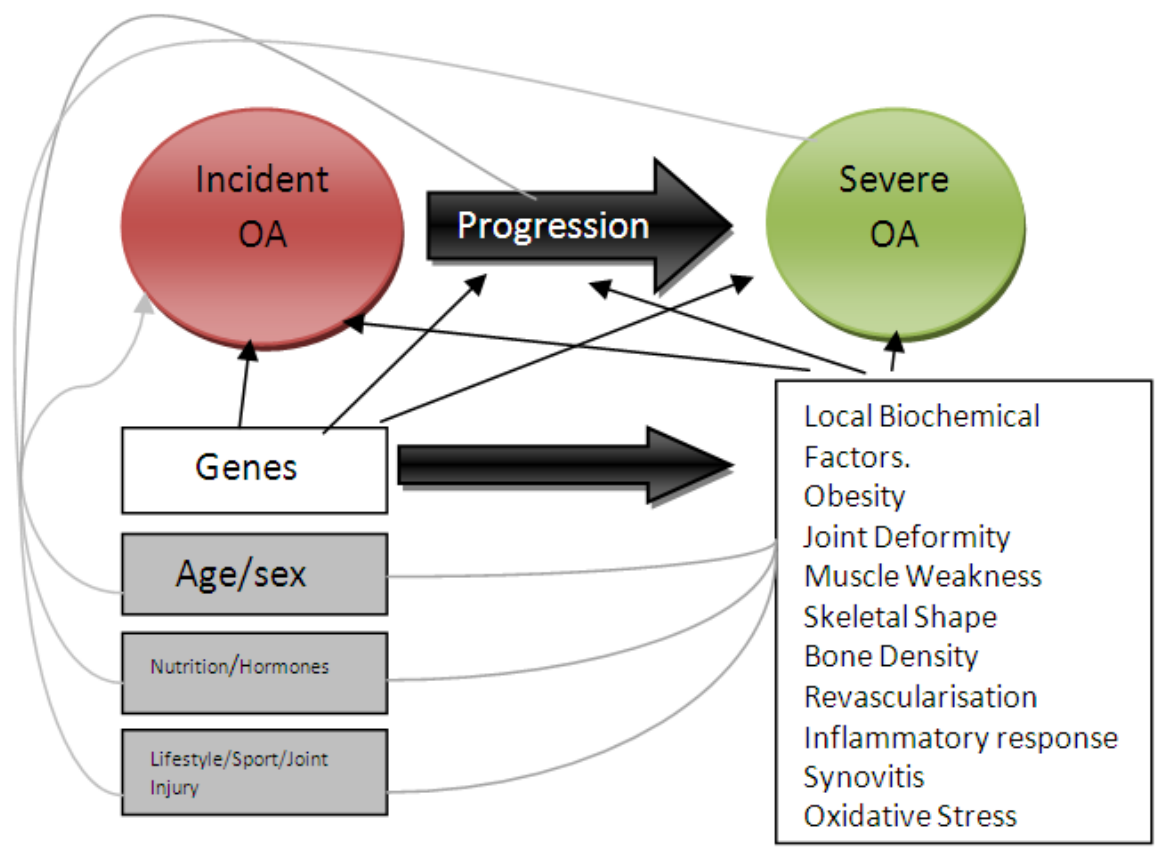

Fig. 1- Mechanisms by which genes can contribute to OA. ${ }^{6}$ 
Heredity plays a role in osteoarthritis as individuals born with other diseases are more likely to develop osteoarthritis. Examples include conditions associated with increased joint laxity (being "double-jointed"), some dysplasia's (abnormal growths of bone and cartilage) and Paget's disease (a type of inflammation in bone that occurs in older people).

3. Smoking: No clear biologic explanation supports an inverse relationship between smoking and OA. However, theories have suggested that smoking may affect the cartilage directly, or when considering knee OA, it may act in an indirect manner by conveying protection through making the subchondral bone more deformable to impact loads. Subsequent epidemiological studies employing the power of the cohort design will complement the results of this study. While these findings corroborate previous studies indicating no association between smoking and OA, anecdotal evidence warrants investigation into the role that cigarette smoking may play in the symptomatology of OA. Future research focusing on the inverse relationship between OA and osteoporosis (known to be positively associated with smoking) may serve to enhance our understanding of the mechanisms affecting the etiology of OA. ${ }^{[7]}$

4. Obesity: Increased body weight, which adds stress to lower body joints, is a well established factor in the development of osteoarthritis. Your knees, which carry the brunt of your weight, are particularly at risk. Fr every pound you gain, you add 4 pounds of pressure on your knees and six times the pressure on your hip. Gaining weight as you head toward middle age can increase the likelihood of developing OA. Recent researc.. suggests that excess body fat produces chemicals that travel throughout the body and cause joint damage, which would mean obesity, plays a systemic, not just a mechanical, role in osteoarthritis onset. ${ }^{[4]}$

Being only 10 pounds overweight increases the force on the knee by $30-60$ pounds with each step. Osteoarthritis is the most common joint disorder with symptoms in the hands, knees, hips, back, and neck. It is unclear exactly how excess weight influences OA. Clearly, being overweight increases the load placed on the joints such as the knee, which increases stress and could possibly hasten the breakdown of cartilage. For example, it is estimated that a force of nearly three to six times one's body weight is exerted across the knee while walking; an increase in body weight increases the force by this amount. However, overweight has also been associated with higher rates of hand $\mathrm{OA}$ in some studies suggesting the involvement of a circulating systemic factor as well.

Overweight women have nearly 4 times the risk of knee $\mathrm{OA}$; for overweight men the risk is 5 times greater. Being overweight is a clear risk factor for developing OA. Population-based studies have consistently shown a link between overweight or obesity and knee OA. Estimating prevalence across populations is difficult since definitions for obesity and knee OA vary among investigators. Data from the first National Health and Nutrition Examination Survey (HANES I) indicated that obese women had nearly 4 times the risk of knee OA as compared with non-obese women; for obese men, the risk was nearly 5 times greater. In a study from Framingham MA, overweight individuals in their thirties who did not have knee OA were at greater risk of later developing the disease. Other investigations, which performed repeated X-rays over time also, have found that being overweight significantly increases the risk of developing knee OA. It is estimated that persons in the highest quintile of body weight have up to 10 times the risk of knee OA than those in the lowest quintile. ${ }^{[8]}$

5. Injury or Overuse: Athletes and people whose jobs require repetitive motion, (landscaping, typing or operating machinery), have a higher risk of developing osteoarthritis due to injury and increased stress on certain joints. Soft tissue injuries, such as ACL tears, can lead to OA; it can also appear in joints affected by previous bone fractures and surgeries. The incidence and prevalence of severe knee injuries requiring medical attention are not well documented. The differential diagnosis of knee injuries includes contusion, subchondral or chondral injury with and without meniscal tear and with and without ligamentous injury or complete tear. In the best studied sports injuries, even injury not precipitating a medical visit appears to be a risk factor for knee OA; the rate is high and increasing worldwide. ${ }^{[4]}$

Meniscus injuries are common in athletes and the general population. Lohmander and colleagues estimate that the cumulative population risk of an anterior cruciate ligament (ACL) injury between 10 and 64 years of age is about $5 \%$ based on MRI findings of the acutely injured knee, and for meniscus injury leading to surgery is at least $15 \%$. The incidence of ACL tears is unknown but the MRI-confirmed incidence has been reported at 81 per 100,000 in a hospital-based study, while a population-based study estimated rates 50 to $100 \%$ higher. The true incidences of both meniscal and ligamentous injuries are likely to be higher since injuries are sometimes unreported, undiagnosed, or diagnosed without MRI.

6. Menopause: OA strikes women more often than men and increases in prevalence, incidence and severity after menopause. Therefore, million dollar questions arises, Is menopause associated with the onset and progression of osteoarthritis in women and can HRT22 render help in such patients. Females are found to have 
more severe OA, more number of joint are involved, have more symptoms and increased hand and knee OA. Many experimental, clinical and epidemiological studies suggest that loss of estrogen at the time of menopause increases a woman's risk of getting osteoarthritis and use of HRT did seem to be associated with not only relieving of symptoms but also reduced rate of progression of osteoarthritis. Moreover, antiresorptive drugs like alendronate may also protect against the development of bone abnormalities associated with knee OA. On the contrary few studies have proposed that estradiol mediates the damage to cartilage tissue and estrogen is chondrodestructive suggesting that HRT is associated with a higher prevalence of clinical OA. Furthermore, polymorphisms in the ER alpha gene have been suggested to be associated with radiographic OA of the knee. Hence with the current level of evidence, HRT cannot be recommended as a first-line treatment against progression of $\mathrm{OA}$, but the fact cannot be denied that if somebody is taking estrogen therapy for some other reason may get benefited. Otherwise treatment will include conventional non-pharmacological and pharmacological treatment.

Fig 2- Levels of physical activity reported by women with arthritis in the US ${ }^{[2]}$

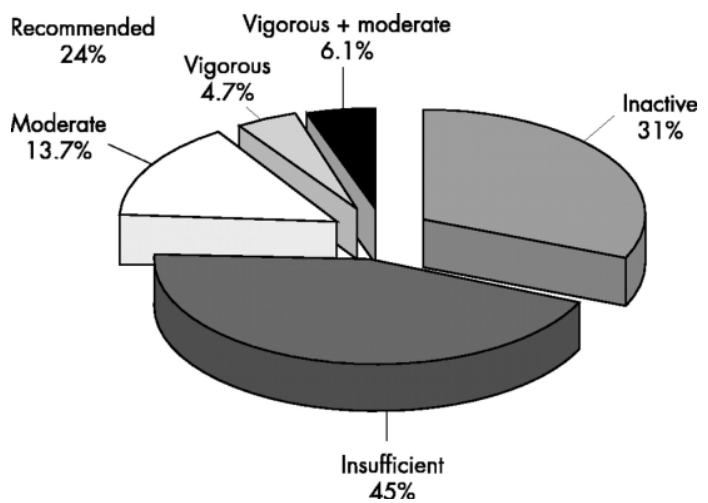

III. Report Of Impending Osteoarthritis: A Case Report.

Figs 3,4,5- These reports of a patient who is on the verge of osteoarthritis explains about the $X$-rays of the spine and femur bones.

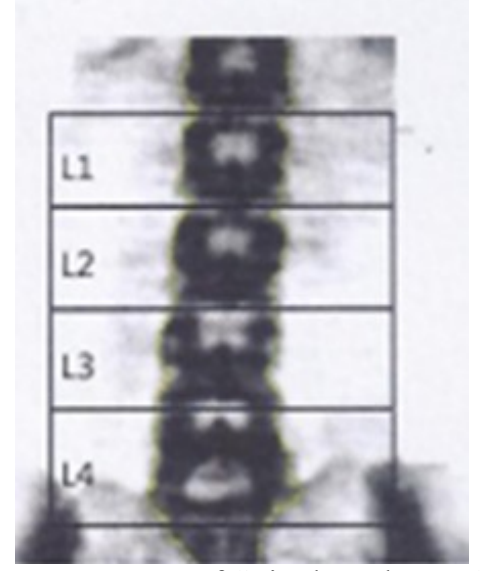

Figure 5-X-ray of Spinal cord

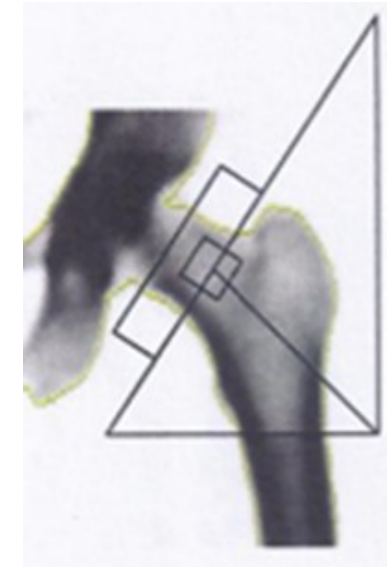

Figure 6- X-ray of left femur

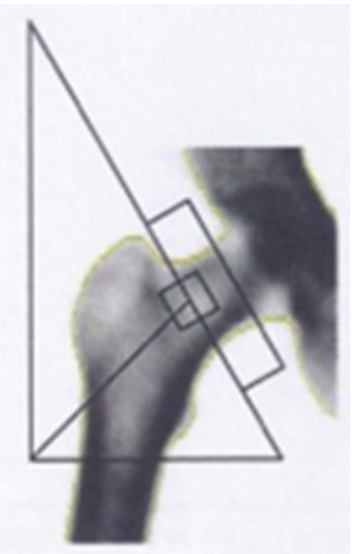

Figure 7- X-ray of right femur

Table: 1

\begin{tabular}{|l|l|l|}
\hline Site & Region & BMD \\
\hline AP Spine & L1-L4 & $0.807 \mathrm{gm} / \mathrm{cm}^{2}$ \\
\hline Dual Femur & Neck left & $0.741 \mathrm{gm} / \mathrm{cm}^{2}$ \\
\hline Dual Femur & Neck right & $0.800 \mathrm{gm} / \mathrm{cm}^{2}$ \\
\hline
\end{tabular}

Since the BMD value of AP spine of the patient is $0.807 \mathrm{~g} / \mathrm{cm}^{2}$ with a T-score of -3.1 the patient is considered osteoarthritis patient according to World Health Organisation (WHO). Fracture risk is high.

The BMD value of left femur bone is $0.740 \mathrm{~g} / \mathrm{cm}^{2}$ with T-score of -1.2 the patient is considered osteoarthritis patient according to World Health Organisation (WHO). Bone density is between $10-25 \%$ below young normal. Fracture risk is moderately low.

The BMD value of right femur bone is $0.800 \mathrm{~g} / \mathrm{cm}^{2}$ with a T-score of -1.7 the patient is considered osteoarthritis patient according to World Health Organisation (WHO). Bone density is around $10-25 \%$ below young normal. Fracture risk is moderately low. 


\section{Prevention.}

Preventing the onset of OA requires lifestyle changes.

1. Primary prevention. These are measures to prevent the condition from occurring. There are only a few effective primary prevention strategies for arthritis. These include:

1.1 Weight control: Obesity is considered a risk factor for OA. Thus, maintaining or reducing weight can lower the risk for certain arthritic conditions.

1.2 Occupational injury prevention: Avoiding repetitive joint use and its injuries can help prevent arthritis.

1.3 Sports injury prevention: Taking the necessary precautions to prevent injury such as warming up and using proper equipment can help reduce joint injuries.

2. Secondary prevention. This involves early diagnosis so that appropriate early intervention can be utilized. However, this is difficult in OA since no effective biomarkers are available to determine the progression of the disease. Furthermore, radiographic evidence is often needed to identify and mark disease progression. Access to health care facilities and availability of X-rays is problematic in many parts of the world.

3. Tertiary prevention. This focuses on reducing the consequences of a disease. Goals of these prevention strategies are to reduce, delay the onset of complications and disability. Tertiary prevention strategies for arthritis are aimed at reducing pain and disability, and improving quality of life. The following encompass tertiary prevention strategies: self-management (weight control, physical activity, education); home help programs; cognitive behavioural interventions; rehabilitation services and medical surgical treatments. ${ }^{[2]}$

\section{Pharmacological treatment.}

\section{Therapeutic Options in Osteoarthritis.}

The American College of Rheumatology (ACR) has issued guidelines for pharmacologic treatment of osteoarthritis of the hand, hip, and knee. For hand osteoarthritis, the ACR conditionally recommends using 1 or more of the following:

Topical capsaicin

Topical nonsteroidal anti-inflammatory drugs (NSAIDs), including trolamine salicylate

Oral NSAIDs

Tramadol

The ACR conditionally recommends against using intra-articular therapies or opioid analgesics for hand osteoarthritis. For patients 75 years and older, the ACR conditionally recommends the use of topical rather than oral NSAIDs.

For knee osteoarthritis, the ACR conditionally recommends using 1 of the following:

Acetaminophen

Oral NSAIDs

Topical NSAIDs

Tramadol

Intra-articular corticosteroid injections

The ACR conditionally recommends against using chondroitin sulfate, glucosamine, or topical capsaicin for knee osteoarthritis. The ACR has no recommendations regarding the use of intra-articular hyaluronates, duloxetine, and opioid analgesics.

For hip osteoarthritis, the ACR conditionally recommends using 1 or more of the following for initial management:

Acetaminophen

Oral NSAIDs

Tramadol

\section{Intra-articular corticosteroid injections.}

The ACR conditionally recommends against using chondroitin sulfate or glucosamine for hip osteoarthritis. The ACR has no recommendation regarding the use of topical NSAIDs, intra-articular hyaluronate injections, duloxetine, or opioid.

\section{Intra-articular injections}

Intra-articular pharmacologic therapy includes injection of a corticosteroid or sodium hyaluronate (ie, hyaluronic acid [HA] or hyaluronan), which may provide pain relief and have an anti-inflammatory effect on the affected joint. Ultrasound guidance can facilitate arthrocentesis and injection and is increasingly being adopted by physicians such as rheumatologists and physiatrists for this purpose. 


\section{Corticosteroid}

After the introduction of the needle into the joint and before steroid administration, aspiration of as much synovial fluid as possible should be attempted. Aspiration often provides symptomatic relief for the patient and allows laboratory evaluation of the fluid, if necessary. Infected joint fluid and bacteremia are contraindications to steroid injection.

In patients with osteoarthritic knee pain, steroid injections generally result in clinically and statistically significant pain reduction as soon as 1 week after injection. The effect may last, on average, anywhere from 4 to 6 weeks per injection, but the benefit is unlikely to continue beyond that time frame. For hip osteoarthritis, a randomized, placebo-controlled study confirmed the effectiveness of corticosteroid injection, with benefits often lasting as long as 3 months.

Some controversial evidence exists regarding frequent steroid injections and subsequent damage to cartilage (chondrodegeneration). Accordingly, it is usually recommended that no more than 3 injections per year be delivered to any individual osteoarthritic joint. Systemic glucocorticoids have no role in the management of osteoarthritis.

\section{Sodium hyaluronate}

Intra-articular injection of sodium hyaluronate, also referred to as viscosupplementation, has been shown to be safe and possibly effective for symptomatic relief of knee osteoarthritis. In the United States, intraarticular HAs are classified as medical devices rather than as drugs.

Intra-articular HAs approved by the FDA for the treatment of osteoarthritic knee pain include the naturally extracted, non-cross-linked sodium hyaluronate products Hyalgan, Supartz, Orthovisc, and Euflexxa, as well as the cross-linked sodium hyaluronate product known as hylan G-F 20 (Synvisc).

Euflexxa is derived from a fermentation process (Streptococcus), whereas the source material for the other products listed is chicken combs. At present, no distinct advantage or disadvantage has been associated with any particular source of HA.

Some differences between the viscosupplements do exist in the FDA-approved prescribing information. For example, whereas Hyalgan and Synvisc have been established as safe for repeat treatment, the safety and efficacy of other products for repeat treatment have not been established.

The exact mechanisms of action through which HAs provide symptomatic relief are unknown. Possible mechanisms include direct binding to receptors (CD44 in particular) in the synovium and cartilage that can lead to several biologic activation pathways.

The HA class in general has demonstrated a very favorable safety profile for chronic pain management in knee osteoarthritis, with the most common adverse event being injection-site pain. Although any intraarticular injection (whether of HAs or of steroids) may elicit an inflammatory response and possible effusion, only the cross-linked hylan G-F 20 product has been associated with a clinically distinct acute inflammatory side effect (ie, severe acute inflammatory reaction [SAIR] or HA-associated intra-articular pseudosepsis).

\section{Prolotherapy}

In a randomized, controlled trial of 90 adults with painful knee osteoarthritis who were randomized to either dextrose prolotherapy, saline injections, or at-home exercise, the patients on prolotherapy experienced significantly greater improvement in pain, function, and stiffness over the other 2 groups. Injections were administered at 1, 5, and 9 weeks, with additional injections provided as needed at weeks 13 and 17 .

\section{Additional pharmacologic agents}

Muscle relaxants may benefit patients with evidence of muscle spasm. Judicious use of narcotics (eg, oxycodone and acetaminophen with codeine) is reserved for patients with severe osteoarthritis.

Glucosamine and chondroitin sulfate have been used in Europe for many years and continue to be popular with patients worldwide. In the United States, however, the glucosamine/chondroitin arthritis intervention trial (GAIT) reported, at best, limited benefit from glucosamine ( $500 \mathrm{mg} 3$ times daily), chondroitin sulfate (400 mg 3 times daily), or the combination of the 2 in patients with knee osteoarthritis. In GAIT patients overall, glucosamine and chondroitin sulfate alone or in combination did not reduce pain effectively at 24 weeks, but in patients with moderate-to-severe pain at baseline, the rate of response was significantly higher with combined therapy than with placebo $(79.2 \%$ vs. $54.3 \%)$. At 2 years, no treatment achieved a clinically important difference in loss of joint-space width, though treatment effects on Kellgren-Lawrence grade 2 knees showed a trend toward improvement relative to the placebo group.

The AHRQ comparison found no clear difference between glucosamine or chondroitin and oral NSAIDs for relieving pain or improving function. However, the AHRQ observed that most trials showing therapeutic benefits from glucosamine used pharmaceutical-grade glucosamine that is not available in the 
United States, noting that the trial findings may therefore be inapplicable to currently available over-the-counter preparations.

Another agent, S-adenosylmethionine (SAM-e), is a European supplement receiving significant attention in the United States. A systematic review of SAM-e found that the evidence was inconclusive, with a number of small trials of questionable quality; the authors concluded that the effects of SAM-e on pain and function may be potentially clinically relevant but are expected to be small.

Chondroprotective drugs (ie, matrix metalloproteinase [MMP] inhibitors and growth factors) are being tested as disease-modifying drugs in the management of osteoarthritis. For example, MMP-13 is specifically expressed in the cartilage of individuals with osteoarthritis but not in the cartilage of normal adults. German researchers reported on the synthesis and biologic evaluation of an MMP-13 selective inhibitor that has demonstrated efficacy as a disease-modifying intra-articular injection for osteoarthritis.

Other investigational agents include monoclonal antibodies that inhibit nerve growth factor (NGF), such as tanezumab. Anti-NGF agents have been shown to reduce chronic pain in patients with osteoarthritis.

\section{Non-Pharmacological Treatments.}

Lifestyle modification, particularly exercise and weight reduction, is a core component in the management of osteoarthritis. Guidelines from Osteoarthritis Research Society International (OARSI) advise that nonpharmacologic treatment of hip and knee osteoarthritis should initially focus on self-help and patientdriven modalities rather than on modalities delivered by health professionals.

The ACR strongly recommends the following nonpharmacologic measures for patients with knee or hip osteoarthritis:

Cardiovascular or resistance land-based exercise

Aquatic exercise

Weight loss, for overweight patients

The ACR conditionally recommends the following measures for patients with knee or hip osteoarthritis:

Self-management programs

Manual therapy in combination with supervised exercise

Psychosocial interventions

Thermal agents

Walking aids, as needed

For patients with knee osteoarthritis, the ACR also conditionally recommends the following measures:

Medially directed patellar taping

Medially wedged insoles for lateral-compartment osteoarthritis

Laterally wedged subtalar strapped insoles for medial-compartment osteoarthritis Tai chi ${ }^{[10]}$

\section{Surgical treatment.}

Surgical treatment of osteoarthritis is usually considered after failure of nonsurgical therapies. There are four surgical procedures: osteotomy, arthroscopy, arthrodesis and arthroplasty. The four procedures have different indications and variable benefits. Total joint arthroplasty, the most surgically advanced in OA treatment, is the mainstay of surgical treatments. Total joint replacement is highly successful and by most measures among the most effective of all medical interventions. Pain, and disability of end-stage OA can be eliminated, restoring patients to near-normal function, and this operation is highly cost-effective. The most common failures of total joint replacement surgery include aseptic loosening and osteolysis, which occur as a result of the corrosion between the implanted material and the cells. Currently there are no RCTs, which have compared surgery with nonsurgical intervention. Although EULAR acknowledges the difficulties in study design, the expert committee recommends that predictors of response, indications for joint replacement, effect of different surgical techniques and long-term effect of joint prosthesis should be examined. Furthermore, postoperative care and outcome assessment should also be studied for these procedures. ${ }^{[2]}$

\section{Platelet-rich Plasma Treatment holds Potential for Treating Osteoarthritis.}

Several treatments for osteoarthritis exist, including exercise, weight control, bracing, nonsteroidal anti-inflammatory, Tylenol, cortisone shots and viscosupplementation, a procedure that involves injecting a gellike substance into the knee to supplement the natural lubricant in the joint. A new treatment that is being studied by a small number of doctors is PRP injections. PRP, which is produced from a patient's own blood, delivers a high concentration of growth factors to arthritic cartilage that can potentially enhance healing. 
Platelet-rich plasma (PRP) holds great promise for treating patients with knee osteoarthritis. The treatment improved pain and function, and in up to $73 \%$ of patients, appeared to delay the progression of osteoarthritis. You take a person's blood, you spin it down, you concentrate the platelets, and you inject a person's knee with their own platelets in a concentrated form. This then activates growth factors and stem cells to help repair the tissue, if possible, calm osteoarthritic symptoms and decrease inflammation. ${ }^{[10]}$

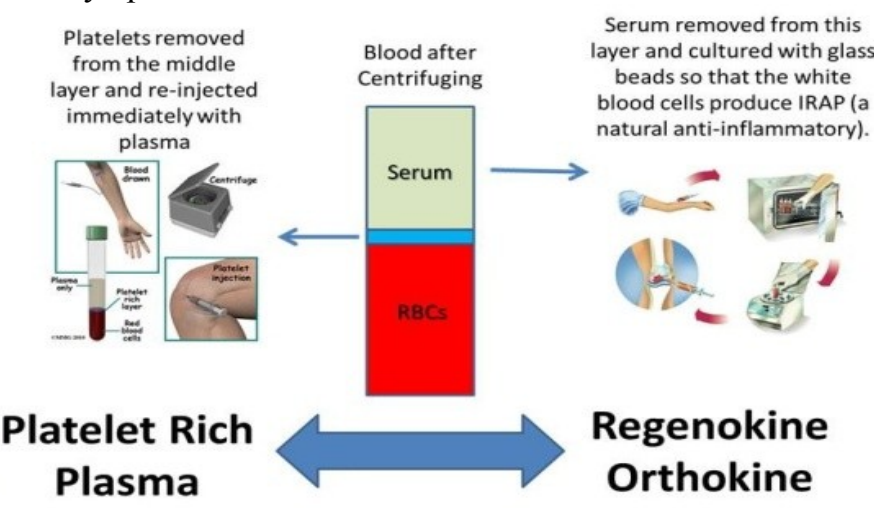

Fig 6- The process of how PRP works

Platelets are crucial for tissue repair and vascular remodelling. The first stage of normal wound healing, immediately following injury or insult, is inflammation, where activated platelets adhere to the site of injury releasing growth factors including:

1. Transforming growth factor (TGF- $\beta$ ): promotes formation of extracellular matrix and regulates bone cell metabolism;

2. Platelet-derived growth factor (PDGF): promotes cell replication, angiogenesis, epithelialisation and granulation tissue formation;

3. Basic fibroblast growth factor (bFGF): promotes proliferation of endothelial cells and fibroblasts and stimulation of angiogenesis;

4. Epidermal growth factor (EGF): promotes cell differentiation and stimulates re-epithelialisation, angiogenesis and collagenase activity;

5. Vascular endothelial growth factor: promotes angiogenesis; and

6. Connective tissue growth factor: promotes angiogenesis, vessel permeability, and stimulates mitogenesis for endothelial cells.

The PRP treatment showed positive effects in patients with knee osteoarthritis. Operated and nonoperated patients showed significant improvement by means of diminishing pain and improved symptoms and quality of life.

Clinical Relevance: There are only a few studies of PRP treatment for cartilage on osteoarthritic knees. Different PRP products might be more or less appropriate to treat different types of tissues and pathologies. The clinical efficacy of PRP remains under debate and a standardized protocol has not yet been established.

\section{Conclusion}

With the cutting edge technologies being developed each passing year that has led to better development and maintenance of human health; the average life span of human being has also increased quite a fold. Living for such long years has its own disadvantages like this progressively degenerative disease, osteoarthritis, one of the most common arthritis known today. The concern part is that there are no proper biomarkers or imaging technology to carefully evaluate and rectify this disease completely. There is still a long way to go for eradicating it. One of the main reasons for this is that since it affects the aged citizens the most, treating them with many chemicals might lead to toxicity build-up in their systems. PRP has proven to be quite a breakthrough in treating osteoarthritis though it is still in the nascent stage. PRP treatment is better than the pharmacological and non-pharmacological treatments that are in current usage since there is no worry of toxic product generation or allergies against those chemicals. PRP thus, should be encouraged among the doctors and more research should be done in this field. OA analysis has a long way to go as we have gathered too much information about the disease but its cure, prevention or treatments are still in their basic stages which still have large potential to improve. 


\section{References}

[1]. Treatment OA Knee, American Academy of Orthopaedic surgeons, 2013

[2]. Saloni Tanna, Pharm.D. MPH, Osteoarthritis "Opportunities to Address Pharmaceutical Gaps", World Health Organisation, 7 October 2004

[3]. http://www.arthritis.org/who-gets-osteoarthritis.php The Arthritis Foundation, 2008

[4]. YongPing Li, XiaoChun Wei, JingMing Zhou, and Lei Wei, The Age-Related Changes in Cartilage and Osteoarthritis, Biomed Res Int. 2013;2013:916530. doi: 10.1155/2013/916530. Epub 2013 Jul 22.

[5]. http://www.webmd.com/osteoarthritis/guide/osteoarthritis-basics The Basics of Osteoarthritis, 2002

[6]. Ana M. Valdes, PhD, Timothy D. Spector, MD, FRCP, The Contribution of Genes to Osteoarthritis, Medical Clinics of North America Volume 93, Issue 1, January 2009, Pages 45-66

[7]. F. V. Wilder, B. J. Hall and J. P. Barrett, Smoking and osteoarthritis: Is there an association? The Clearwater Osteoarthritis Study, Elsevier, Osteoarthritis Cartilage, 11(1):29-35, 2003 Jan

[8]. Susan Bartlett, Ph.D., Role of Body Weight in Osteoarthritis, Johns Hopkins Rheumatology, March 27, 2012

[9]. Carlos J Lozada, MD; Chief Editor: Herbert S Diamond, MD, Osteoarthritis Treatment \& Management, Updated: Sep 2, 2014

[10]. Platelet-rich Plasma (PRP) Treatment Shows Potential for Knee Osteoarthritis, Hospital for Special Surgery, February $12,2013$. 